\title{
Dynamic Nulling-and-Canceling for Efficient Near-ML Decoding of MIMO Systems
}

\author{
Dominik Seethaler, Student Member, IEEE, Harold Artés, Member, IEEE, and \\ Franz Hlawatsch, Senior Member, IEEE
}

\begin{abstract}
It is known that conventional nulling-and-canceling (NC) detection for multiple-input/multiple-output (MIMO) systems cannot exploit all of the available diversity, and, thus, its performance is significantly inferior to that of maximum likelihood (ML) detection. Conventional NC employs the layerwise postequalization signal-to-noise ratios (SNRs) as reliability measures for layer sorting. These SNRs are average quantities that do not depend on the received vector. In this paper, we propose the novel dynamic nulling-and-canceling (DNC) technique that uses approximate a posteriori probabilities as measures of layer reliability. The DNC technique is a minimum mean-square error (MMSE) nulling scheme combined with an improved "dynamic" layer sorting rule that exploits the information contained in the current received vector. We calculate the error probability of DNC for a simple special case and show that it is upper bounded by the error probability of conventional NC. Simulation results are presented for spatial multiplexing systems and for systems using linear dispersion codes. It is demonstrated that the DNC technique can yield near-ML performance for a wide range of system sizes and channel SNRs at a fraction of the computational complexity of the sphere-decoding algorithm for ML detection.
\end{abstract}

Index Terms-Equalization, linear dispersion codes, maximum likelihood (ML) detection, multiple-input/multiple-output (MIMO) channels, multiuser detection, nulling and canceling (NC), spatial multiplexing, V-BLAST.

\section{INTRODUCTION}

$\mathbf{F}$ OR digital communications over wireless fading channels, multiple-input/multiple-output (MIMO) systems offer the potential of large data rates and high reliability. The reliability advantage of MIMO systems is due to the inherently available diversity (e.g., [3]), which, however, cannot be exploited equally well by the various existing detection schemes. Maximum-likelihood (ML) detection exploits all of the available diversity but tends to have high computational complexity. This is also the case for the sphere-decoding algorithm [4] for ML detection. On the other hand, suboptimum detection schemes like equalization-based techniques (e.g., [5]) and nulling-and-canceling (NC) or decision-feedback techniques [5]-[7] are much less

Manuscript received January 20, 2005; revised December 28, 2005. This work was supported in part by the FWF by Grant P15156 and by the Max Kade Foundation, NY. Parts of this work have been presented at the International Conference on Acoustics, Speech, and Signal Processing, Montreal, QC, Canada, May 2004, and EUSIPCO, Antalya, Turkey, September 2005. The associate editor coordinating the review of this manuscript and approving it for publication was Prof. Javier Garcia-Frias.

D. Seethaler and F. Hlawatsch are with the Institute of Communications and Radio-Frequency Engineering, Vienna University of Technology, A-1040 Vienna, Austria (e-mail: dominik.seethaler@tuwien.ac.at).

H. Artés is with the Information Systems Laboratory, Stanford University, Stanford, CA 94305 USA.

Digital Object Identifier 10.1109/TSP.2006.881195 complex but their performance is significantly inferior to that of ML detection. Thus, there is a strong demand for computationally efficient suboptimum detection techniques that exploit a large part of the available diversity and whose performance can hence come close to that of ML detection.

\section{A. Nulling-and-Canceling With "Dynamic" Layer Sorting}

An attractive suboptimum detection technique is NC with layer sorting using the layerwise postequalization signal-to-noise ratios (PSNRs) as a reliability criterion [8]. The performance of this technique is much better than that of equalization techniques or of $\mathrm{NC}$ without layer sorting, although it is still far inferior to that of ML detection. It is interesting to observe that the PSNRs are just average quantities that do not depend on the received vector-more specifically, they depend on the channel realization and on the mean noise power, but neither on the transmitted data vector nor on the noise realization.

In this paper, we propose an improved $\mathrm{NC}$ technique [1] that we term dynamic nulling-and-canceling (DNC) because it explicitly takes into account the current received vector. The basic idea of the DNC scheme is to use the a posteriori probability (APP) of each layer as a measure of layer reliability. To keep the complexity low, we use an approximate APP that is constructed by means of a Gaussian approximation for the residual postequalization interference. This approach is inspired and motivated by [9], where an iteratively updated Gaussian approximation for the postequalization interference was used in the context of multiuser detection. As we will show, the Gaussian approximation approach results in a minimum mean-square error (MMSE) equalization based nulling technique with a simple layer-sorting rule that is "dynamic" in that it depends on the current received vector, in contrast to the "static" (average) layer sorting employed by conventional NC. We will demonstrate that this technique can yield near-ML performance for a wide range of system sizes and channel signal-to-noise ratios (SNRs).

Dynamic layer sorting is more complex than conventional layer sorting because the layers are sorted anew for each received vector and not just for an entire data block during which the channel is constant. To keep the extra complexity small, we use an efficient recursive technique for matrix inversion that was proposed for NC in [10]. The computational complexity of the resulting efficient DNC implementation is only a fraction of that required by the sphere-decoding algorithm for ML detection.

\section{B. Paper Structure}

In the remainder of this section, we describe the system model and briefly review existing MIMO detection techniques with 
emphasis on NC-based data detection. In Section II, we formulate the basic principle of the novel DNC technique. The two stages of DNC - data detection and layer sorting - are then developed in Section III and Section IV, respectively. In Section V, a statement of the overall DNC algorithm is provided, an efficient method for recursive matrix inversion is discussed, and the algorithm's computational complexity is analyzed. The error performance of DNC is studied for a simple special case in Section VI. Finally, in Section VII we assess the symbol-error-rate performance and computational complexity of DNC through numerical simulations carried out for spatial multiplexing systems and for systems using linear dispersion codes.

\section{System Model}

We assume a linear MIMO model where the transmitted data vector $\mathbf{d} \triangleq\left(d_{1} \cdots d_{M}\right)^{T}$ of size $M$ and the received vector $\mathbf{r} \triangleq\left(r_{1} \cdots r_{N}\right)^{T}$ of size $N \geq M$ are related according to

$$
\mathbf{r}=\mathbf{H d}+\mathbf{w} \text {. }
$$

Here, the $N \times M$ matrix $\mathbf{H}$ denotes the system matrix and $\mathbf{w} \triangleq$ $\left(w_{1} \cdots w_{N}\right)^{T}$ denotes the noise vector. The data vector components $d_{k}(k \in\{1, \ldots, M\}$ indexes the layer $)$ are drawn from a complex symbol alphabet $\mathcal{A}$ and are assumed zero-mean and independent with unit variance. The noise components $w_{i}$ are assumed independent and circularly symmetric complex Gaussian with variance $\sigma_{w}^{2}$. The system matrix $\mathbf{H}$ is supposed to be perfectly known at the receiver.

Several different space-time transmission schemes can be written in the form of the linear MIMO model (1). For a spatial multiplexing system such as V-BLAST [5], [6], the $k$ th data symbol $d_{k}$ is directly transmitted on the $k$ th transmit antenna; $M$ and $N$ represent the number of transmit antennas and the number of receive antennas, respectively; and the system matrix $\mathbf{H}$ is the MIMO channel matrix (i.e., the elements of $\mathbf{H}$ are the fading coefficients from a transmit antenna to a receive antenna). Other space-time transmission schemes that can be described by the model (1) include linear dispersion codes [11], where (1) is obtained through linear coding of the data streams over space and consecutive channel uses, and unitary differential space-time modulation using the Cayley transform [12], where (1) correponds to the "linearized" system model that is obtained without explicit estimation of the MIMO channel coefficients.

\section{Review of Detection Techniques for MIMO Systems}

The major detection techniques for MIMO systems are linear equalization followed by quantization (e.g., [5]), NC (also known as decision-feedback) [5]-[7], and ML detection [4], [13]. The first two techniques are suboptimum but significantly less complex than ML detection. As a relevant background and for reference in subsequent sections, we will briefly review these three detection techniques.

1) Linear Equalization Based Detection: In linear equalization based detection, an estimate of the transmitted data vector $\mathbf{d}$ is calculated as $\mathbf{y}=\mathbf{G r}$ where $\mathbf{G}$ is an equalizer matrix. The detected data vector $\hat{\mathbf{d}}$ is then obtained by layerwise quantization according to the symbol alphabet $\mathcal{A}$, i.e.,

$$
\hat{d}_{k}=Q\left\{y_{k}\right\} \triangleq \underset{a \in \mathcal{A}}{\arg \min }\left|y_{k}-a\right|^{2}
$$

In general, layerwise quantization is suboptimum because it ignores the correlations of the noise components introduced by the multiplication of $\mathbf{r}$ by $\mathbf{G}$.

The zero-forcing $(\mathrm{ZF})$ equalizer is given by the pseudo-inverse [14] of $\mathbf{H}$, i.e., $\mathbf{G}=\mathbf{H}^{\#}=\left(\mathbf{H}^{H} \mathbf{H}\right)^{-1} \mathbf{H}^{H}$. (For the last expression, we assumed that $N \geq M$ and that $\mathbf{H}$ has full rank.) Thus, the result of $\mathrm{ZF}$ equalization-before quantization-is

$$
\mathbf{y}_{\mathrm{ZF}}=\mathbf{H}^{\#} \mathbf{r}=\left(\mathbf{H}^{H} \mathbf{H}\right)^{-1} \mathbf{H}^{H} \mathbf{r}=\mathbf{d}+\tilde{\mathbf{w}}
$$

which is the transmitted data vector $\mathbf{d}$ corrupted by correlated noise $\tilde{\mathbf{w}}=\mathbf{H}^{\#} \mathbf{w}$ with covariance matrix

$$
\mathbf{R}_{\tilde{\mathbf{w}}}=\sigma_{w}^{2}\left(\mathbf{H}^{H} \mathbf{H}\right)^{-1} .
$$

The MMSE equalizer is given by $\mathbf{G}=\left(\mathbf{H}^{H} \mathbf{H}+\sigma_{w}^{2} \mathbf{I}\right)^{-1} \mathbf{H}^{H}$, which minimizes the mean-square error (MSE) $\mathrm{E}\left\{\|\mathbf{y}-\mathbf{d}\|^{2}\right\}$ [15]. Thus, the result of MMSE equalization is

$$
\mathbf{y}_{\mathrm{MMSE}}=\left(\mathbf{H}^{H} \mathbf{H}+\sigma_{w}^{2} \mathbf{I}\right)^{-1} \mathbf{H}^{H} \mathbf{r} .
$$

2) $N C$ : NC uses a serial decision-feedback approach to detect the layers one after another (e.g., [5]-[7]). At the first decoding step, ZF or MMSE equalization based detection is applied to a certain layer $k_{1} \in\{1, \ldots, M\}$, yielding $\hat{d}_{k_{1}}=Q\left\{(\mathbf{G r})_{k_{1}}\right\}$. Then, the interference corresponding to $\hat{d}_{k_{1}}$ is subtracted from $\mathbf{r}$, i.e.,

$$
\mathbf{r}^{(2)}=\mathbf{r}-(\mathbf{H})_{k_{1}} \hat{d}_{k_{1}},
$$

where $(\mathbf{H})_{k_{1}}$ denotes the $k_{1}$ th column of the system matrix $\mathbf{H}$. If the decision $\hat{d}_{k_{1}}$ was correct, i.e., $\hat{d}_{k_{1}}=d_{k_{1}}$, we obtain the reduced system model

$$
\mathbf{r}^{(2)}=\mathbf{H}^{(2)} \mathbf{d}^{(2)}+\mathbf{w}
$$

Here, the reduced system matrix $\mathbf{H}^{(2)}$ of size $N \times(M-1)$ is the system matrix $\mathbf{H}$ with the $k_{1}$ th column removed, and the reduced data vector $\mathbf{d}^{(2)}$ of size $M-1$ is the data vector d with the $k_{1}$ th component removed. At the second decoding step, we perform a re-indexing of the remaining layers, i.e., $\{1, \ldots, M\} \backslash\left\{k_{1}\right\} \rightarrow\{1, \ldots, M-1\}$, and we detect a specific layer $d_{k_{2}}^{(2)}$ with $k_{2} \in\{1, \ldots, M-1\}$. This detection is based on the reduced system model in (5), i.e., $\hat{d}_{k_{2}}^{(2)}=Q\left\{\left(\mathbf{G}^{(2)} \mathbf{r}^{(2)}\right)_{k_{2}}\right\}$, where $\mathbf{G}^{(2)}$ denotes the ZF or MMSE equalizer corresponding to $\mathbf{H}^{(2)}$. Subsequently, the interference corresponding to $\hat{d}_{k_{2}}^{(2)}$ is subtracted from $\mathbf{r}^{(2)}$. This detection-subtraction procedure is repeated until all $M$ layers are detected.

The performance of NC depends crucially on the order of the layers $k_{1}, \ldots, k_{M}$. To minimize error propagation effects and to optimally support the processing of unreliable layers by means of the additional degrees of diversity that become available in the reduced system models, more reliable layers should be detected first. Therefore, the layers are commonly ordered (sorted) using the layerwise ZF or MMSE PSNRs as measures of layer 
reliability [5], [8]. NC with layer sorting significantly outperforms NC without layer sorting, although it is still far inferior to ML detection (see Section VII).

3) ML Detection: ML vector detection is optimum in the sense of minimum vector error probability when all data vectors are equally likely. For our system model (1) and our assumptions in Section I-C, the ML detector is given by

$$
\hat{\mathbf{d}}_{\mathrm{ML}}=\underset{\mathbf{d} \in \mathcal{D}}{\arg \min }\|\mathbf{r}-\mathbf{H d}\|^{2} .
$$

Here, $\mathcal{D}$ denotes the set of all data vectors $\mathbf{d}$. We note that $\mathcal{D}=$ $\mathcal{A}^{M}$, where $\mathcal{A}$ is the symbol alphabet, and thus $|\mathcal{D}|=|\mathcal{A}|^{M}$. Hence, the computational complexity of ML detection grows exponentially with $M$. As shown in [16], the same is true for the average complexity of the sphere-decoding algorithm [4] for ML detection, although for sufficiently large SNR the average complexity of sphere-decoding behaves polynomially in $M$ as long as $M$ is not too large [17].

\section{Fundamentals OF DNC}

We now describe the basic principle of the novel DNC technique, namely, the construction of the approximate APP and its use for symbol detection and "dynamic" layer sorting. We will consider just the first decoding step, where we detect a layer $k_{1}$ in favor of a symbol $\hat{d}_{k_{1}}$ with high reliability. Layer $k_{1}$ is then used to perform the interference cancelation as in conventional NC (see Section I-D). The subsequent detection and interference-cancelation steps are analogous, however with a reduced number of active layers. A complete statement of the DNC algorithm will be provided in Section V.

\section{A. MAP Approach to Detection and Layer Sorting}

For the $k$ th layer, $k \in\{1, \ldots, M\}$, the optimum decision on the data symbol $d_{k} \in \mathcal{A}$ is given by the maximum a posteriori (MAP) rule that maximizes the $\mathrm{APP}^{1} \mathrm{P}\left[d_{k}=a \mid \mathbf{y}_{\mathrm{ZF}}\right]$ [13]:

$$
\hat{d}_{k} \triangleq \underset{a \in \mathcal{A}}{\arg \max } \mathrm{P}\left[d_{k}=a \mid \mathbf{y}_{\mathrm{ZF}}\right]
$$

The resulting maximum APP $\mathrm{P}\left[d_{k}=\hat{d}_{k} \mid \mathbf{y}_{\mathrm{ZF}}\right]$ characterizes the reliability of the symbol decision $\hat{d}_{k}$. Our approach to layer sorting now is to first calculate the optimum symbol $\hat{d}_{k}$ for each layer $k$ and then choose the layer $k$ for which the reliability of this optimum symbol decision (APP for $d_{k}=\hat{d}_{k}$ ) is maximum, i.e.,

$$
k_{1} \triangleq \underset{k \in\{1, \ldots, M\}}{\arg \max } \mathrm{P}\left[d_{k}=\hat{d}_{k} \mid \mathbf{y}_{\mathrm{ZF}}\right]
$$

Decoding layer $k_{1}$ in favor of $\hat{d}_{k_{1}}$ has maximum reliability at this stage, and we subsequently use this result for interference cancelation.

The complexity of calculating the APP $\mathrm{P}\left[d_{k}=a \mid \mathbf{y}_{\mathrm{ZF}}\right]$ required in (6) is exponential in $M$. A significant reduction of

\footnotetext{
${ }^{1}$ The APP can equivalently be conditioned on the result of ZF equalization $\mathbf{y}_{\mathrm{ZF}}=\mathbf{H}^{\#} \mathbf{r}$ (see (3)) rather than on the received vector $\mathbf{r}$ since ZF equalization without quantization does not imply any loss of information.
}

complexity can be obtained by an approximation. We first observe that by Bayes' rule, the APP can be rewritten in terms of the conditional probability density function (pdf) $f\left(\mathbf{y}_{\mathrm{ZF}} \mid d_{k}=\right.$ a) as

$$
\mathrm{P}\left[d_{k}=a \mid \mathbf{y}_{\mathrm{ZF}}\right]=\frac{f\left(\mathbf{y}_{\mathrm{ZF}} \mid d_{k}=a\right)}{\sum_{a^{\prime} \in \mathcal{A}} f\left(\mathbf{y}_{\mathrm{ZF}} \mid d_{k}=a^{\prime}\right)}
$$

where it has been assumed that all data symbols are transmitted equally likely, i.e., $\mathrm{P}\left[d_{k}=a\right]=1 /|\mathcal{A}|$ for all $a \in \mathcal{A}$. We have

$$
f\left(\mathbf{y}_{\mathrm{ZF}} \mid d_{k}=a\right) \propto \sum_{\mathbf{d} \in \mathcal{D}(a, k)} f\left(\mathbf{y}_{\mathrm{ZF}} \mid \mathbf{d}\right)
$$

where $\mathcal{D}(a, k)$ denotes the set of all $\mathbf{d} \in \mathcal{D}$ for which $d_{k}=a$. Because according to (3) $f\left(\mathbf{y}_{\mathrm{ZF}} \mid \mathbf{d}\right)$ is a Gaussian pdf, it follows from (9) that $f\left(\mathbf{y}_{\mathrm{ZF}} \mid d_{k}=a\right)$ is a Gaussian mixture pdf.

Under the condition that $d_{k}=a$, we can reformulate $\mathbf{y}_{\mathrm{ZF}}=$ $\mathbf{d}+\tilde{\mathbf{w}}$ in (3) as

$$
\mathbf{y}_{\mathrm{ZF}}=a \mathbf{e}_{k}+\sum_{\substack{j=1 \\ j \neq k}}^{M} d_{j} \mathbf{e}_{j}+\tilde{\mathbf{w}}, \quad \text { for } d_{k}=a
$$

where $\mathbf{e}_{i}$ denotes the $i$ th $M$-dimensional unit vector. This shows that for $d_{k}=a, \mathbf{y}_{\mathrm{ZF}}$ is equal (up to a shift by $a \mathbf{e}_{k}$ ) to the postequalization interference for the $k$ th layer, $\sum_{j \neq k} d_{j} \mathbf{e}_{j}+\tilde{\mathbf{w}}$.

\section{B. Gaussian Approximation}

We now use a Gaussian approximation for the postequalization interference to obtain a computationally efficient approximation to (8). More specifically, we approximate the Gaussian mixture pdf $f\left(\mathbf{y}_{\mathrm{ZF}} \mid d_{k}=a\right)$ by the Gaussian pdf $^{2}$

$$
\tilde{f}_{k}\left(\mathbf{y}_{\mathrm{ZF}} \mid d_{k}=a\right) \triangleq \frac{1}{\pi^{M} \operatorname{det}\left(\mathbf{C}_{k}\right)} e^{-\left(\mathbf{y}_{\mathrm{ZF}}-\boldsymbol{\mu}_{k}\right)^{H} \mathbf{C}_{k}^{-1}\left(\mathbf{y}_{\mathrm{ZF}}-\boldsymbol{\mu}_{k}\right)} .
$$

Here, the mean $\boldsymbol{\mu}_{k}$ and the covariance $\mathbf{C}_{k}$ are chosen consistent with the true pdf $f\left(\mathbf{y}_{\mathrm{ZF}} \mid d_{k}=a\right)$, i.e., $\boldsymbol{\mu}_{k} \triangleq \mathrm{E}\left[\mathbf{y}_{\mathrm{ZF}} \mid d_{k}=a\right]$ and $\mathbf{C}_{k} \triangleq \operatorname{cov}\left[\mathbf{y}_{\mathrm{ZF}} \mid d_{k}=a\right]$. From (10), we obtain

$$
\boldsymbol{\mu}_{k}=a \mathbf{e}_{k}, \quad \mathbf{C}_{k}=\mathbf{I}-\mathbf{e}_{k} \mathbf{e}_{k}^{T}+\mathbf{R}_{\tilde{\mathbf{w}}} .
$$

The Gaussian pdf $\tilde{f}_{k}\left(\mathbf{y}_{\mathrm{ZF}} \mid d_{k}=a\right)$ is now completely determined, and the APP in (8) is approximated by

$$
\mathrm{P}\left[d_{k}=a \mid \mathbf{y}_{\mathrm{ZF}}\right] \approx \frac{\tilde{f}_{k}\left(\mathbf{y}_{\mathrm{ZF}} \mid d_{k}=a\right)}{\sum_{a^{\prime} \in \mathcal{A}} \tilde{f}_{k}\left(\mathbf{y}_{\mathrm{ZF}} \mid d_{k}=a^{\prime}\right)} .
$$

Using this Gaussian approximation, the maximization in (6) that yielded the optimum symbol for the $k$ th layer is replaced by

$$
\begin{aligned}
\hat{d}_{k} & \triangleq \underset{a \in \mathcal{A}}{\arg \max }\left\{\frac{\tilde{f}_{k}\left(\mathbf{y}_{\mathrm{ZF}} \mid d_{k}=a\right)}{\sum_{a^{\prime} \in \mathcal{A}} \tilde{f}_{k}\left(\mathbf{y}_{\mathrm{ZF}} \mid d_{k}=a^{\prime}\right)}\right\} \\
& =\underset{a \in \mathcal{A}}{\arg \max } \tilde{f}_{k}\left(\mathbf{y}_{\mathrm{ZF}} \mid d_{k}=a\right) .
\end{aligned}
$$

${ }^{2}$ We assume that $\mathbf{C}_{k}=\mathbf{I}-\mathbf{e}_{k} \mathbf{e}_{k}^{T}+\mathbf{R}_{\tilde{\mathbf{w}}}$ is nonsingular (this is guaranteed if $\sigma_{w}^{2} \neq 0$ and $\mathbf{H}$ has full rank). 
Furthermore, the maximization in (7) that yielded the optimum layer is replaced by 3

$$
k_{1} \triangleq \underset{k \in\{1, \ldots, M\}}{\arg \max }\left\{\frac{\tilde{f}_{k}\left(\mathbf{y}_{\mathrm{ZF}} \mid d_{k}=\hat{d}_{k}\right)}{\sum_{a \in \mathcal{A}} \tilde{f}_{k}\left(\mathbf{y}_{\mathrm{ZF}} \mid d_{k}=a\right)}\right\} .
$$

In what follows, these new definitions for $\hat{d}_{k}$ and $k_{1}$ will be used rather than the original definitions (6) and (7).

Equations (13) and (14) define, respectively, the data-detection stage and layer-sorting stage of DNC. Next, we will develop the calculations corresponding to these two stages.

\section{DATA-Detection STAGe}

Using the Gaussian approximation (11), (12), we can write the approximate MAP decision (13) as

$$
\begin{aligned}
\hat{d}_{k} & =\underset{a \in \mathcal{A}}{\arg \max }\left\{\frac{1}{\pi^{M} \operatorname{det}\left(\mathbf{C}_{k}\right)} e^{-\left(\mathbf{y}_{\mathrm{ZF}}-a \mathbf{e}_{k}\right)^{H} \mathbf{C}_{k}^{-1}\left(\mathbf{y}_{\mathrm{ZF}}-a \mathbf{e}_{k}\right)}\right\} \\
& =\underset{a \in \mathcal{A}}{\arg \max }\left\{-\left(\mathbf{y}_{\mathrm{ZF}}-a \mathbf{e}_{k}\right)^{H} \mathbf{C}_{k}^{-1}\left(\mathbf{y}_{\mathrm{ZF}}-a \mathbf{e}_{k}\right)\right\} \\
& =\underset{a \in \mathcal{A}}{\arg \max }\left\{2 \operatorname{Re}\left\{\mathbf{y}_{\mathrm{ZF}}^{H} \mathbf{C}_{k}^{-1} \mathbf{e}_{k} a\right\}-|a|^{2} \mathbf{e}_{k}^{T} \mathbf{C}_{k}^{-1} \mathbf{e}_{k}\right\} .
\end{aligned}
$$

The matrix inversion lemma [14] applied to $\mathbf{C}_{k}^{-1}=\left(\mathbf{I}-\mathbf{e}_{k} \mathbf{e}_{k}^{T}+\right.$ $\left.\mathbf{R}_{\tilde{\mathbf{w}}}\right)^{-1}$ yields

$$
\mathbf{C}_{k}^{-1}=\mathbf{W}\left(\mathbf{I}+\frac{\mathbf{e}_{k} \mathbf{e}_{k}^{T} \mathbf{W}}{1-W_{k, k}}\right)
$$

with

$$
\mathbf{W} \triangleq\left(\mathbf{I}+\mathbf{R}_{\tilde{\mathbf{w}}}\right)^{-1}=\left(\mathbf{I}+\sigma_{w}^{2}\left(\mathbf{H}^{H} \mathbf{H}\right)^{-1}\right)^{-1}
$$

and with $W_{k, k} \in \mathbb{R}$ denoting the $k$ th diagonal element of $\mathbf{W}$. The $M \times M$ matrix $\mathbf{W}$ is termed Wiener estimator in [18]; it converts ZF equalization (3) into MMSE equalization (4) [18], i.e.,

$$
\mathbf{W y}_{\mathbf{Z F}}=\mathbf{y}_{\mathrm{MMSE}} \cdot
$$

Using this result and (16), we obtain

$$
\begin{aligned}
\mathbf{y}_{\mathrm{ZF}}^{H} \mathbf{C}_{k}^{-1} \mathbf{e}_{k} & =\mathbf{y}_{\mathrm{ZF}}^{H} \mathbf{W} \mathbf{e}_{k}\left(1+\frac{\mathbf{e}_{k}^{T} \mathbf{W} \mathbf{e}_{k}}{1-W_{k, k}}\right) \\
& =y_{\mathrm{MMSE}, k}^{*}\left(1+\frac{W_{k, k}}{1-W_{k, k}}\right) \\
& =\frac{y_{\mathrm{MMSE}, k}^{*}}{1-W_{k, k}}
\end{aligned}
$$

and

$$
\begin{aligned}
\mathbf{e}_{k}^{T} \mathbf{C}_{k}^{-1} \mathbf{e}_{k} & =\mathbf{e}_{k}^{T} \mathbf{W} \mathbf{e}_{k}\left(1+\frac{\mathbf{e}_{k}^{T} \mathbf{W} \mathbf{e}_{k}}{1-W_{k, k}}\right) \\
& =W_{k, k}\left(1+\frac{W_{k, k}}{1-W_{k, k}}\right) \\
& =\frac{W_{k, k}}{1-W_{k, k}} .
\end{aligned}
$$

\footnotetext{
${ }^{3}$ Whereas the denominator in (8), $\sum_{a \in \mathcal{A}} f\left(\mathbf{y}_{\mathrm{ZF}} \mid d_{k}=a\right)=|\mathcal{A}| f\left(\mathbf{y}_{\mathrm{ZF}}\right)$, does not depend on the layer index $k$, the denominator in (14), $\sum_{a \in \mathcal{A}} \tilde{f}_{k}\left(\mathbf{y}_{\mathrm{ZF}} \mid d_{k}=a\right)=|\mathcal{A}| \tilde{f}_{k}\left(\mathbf{y}_{\mathrm{ZF}}\right)$, depends on $k$.
}

Thus, the maximization in (15) simplifies to

$$
\hat{d}_{k}=\underset{a \in \mathcal{A}}{\arg \max }\left\{\frac{1}{1-W_{k, k}}\left(2 \operatorname{Re}\left\{y_{\mathrm{MMSE}, k}^{*} a\right\}-W_{k, k}|a|^{2}\right)\right\} .
$$

The eigenvalues $\lambda_{W, k}$ of $\mathbf{W}$ satisfy $0 \leq \lambda_{W, k}<1$. Because $W_{k, k}$ can be written as a quadratic form induced by $\mathbf{W}$ and this quadratic form is bounded by the minimum and maximum eigenvalues of $\mathbf{W}$, we obtain the following two (equivalent) inequalities:

$$
0 \leq W_{k, k}<1, \quad 1 \leq \frac{1}{1-W_{k, k}}<\infty .
$$

Assuming that $W_{k, k} \neq 0$ for $k=1, \ldots, M$ (which holds if $\mathbf{H}$ has full rank), we can rewrite (20) as

$$
\begin{aligned}
\hat{d}_{k} & =\underset{a \in \mathcal{A}}{\arg \max }\left\{\frac{W_{k, k}}{1-W_{k, k}}\left(2 \frac{\operatorname{Re}\left\{y_{\mathrm{MMSE}, k}^{*} a\right\}}{W_{k, k}}-|a|^{2}\right)\right\} \\
& =\underset{a \in \mathcal{A}}{\arg \min }\left\{\frac{\left|y_{\mathrm{MMSE}, k}\right|^{2}}{W_{k, k}^{2}}-2 \frac{\operatorname{Re}\left\{y_{\mathrm{MMSE}, k}^{*} a\right\}}{W_{k, k}}+|a|^{2}\right\} \\
& =\underset{a \in \mathcal{A}}{\arg \min } \psi_{k}^{2}(a)
\end{aligned}
$$

with the "unbiased distance"

$$
\psi_{k}(a) \triangleq\left|\frac{y_{\mathrm{MMSE}, k}}{W_{k, k}}-a\right| .
$$

The minimization in (21) is known as unbiased MMSE detection [19]; it will hereafter be denoted as

$$
\hat{d}_{k}=Q_{\mathrm{u}}\left\{y_{\mathrm{MMSE}, k}\right\} .
$$

The terms "unbiased distance" and "unbiased MMSE detection" reflect the fact that, in contrast to the MMSE estimate $y_{\mathrm{MMSE}, k}$, the scaled MMSE estimate $y_{\mathrm{MMSE}, k} / W_{k, k}$ is conditionally unbiased given $d_{k}$, i.e., $\mathrm{E}\left\{y_{\mathrm{MMSE}, k} / W_{k, k}-d_{k} \mid d_{k}\right\}=0$ or equivalently $\mathrm{E}\left\{\left(y_{\mathrm{MMSE}, k} / W_{k, k}\right) \mid d_{k}\right\}=d_{k}$ [19]. In general, the error probability of unbiased MMSE detection (23) is slightly smaller than that of conventional MMSE detection. (Recall that for conventional MMSE detection, each component $y_{\mathrm{MMSE}, k}$ of the MMSE-equalized received vector $\mathbf{y}_{\mathrm{MMSE}}$ is quantized according to (2).) Unbiased and conventional MMSE detection are however fully equivalent for constant-modulus signaling, i.e., when $|a|$ is equal for all symbols $a \in \mathcal{A}$.

Thus, our development in this section has shown that the data-detection stage of DNC - that is, approximate MAP detection using the Gaussian approximation for the postequalization interference-is equivalent to unbiased MMSE detection, which is computationally simple.

\section{LAYER-SORTING STAGE}

We now develop the calculations corresponding to the layersorting stage. This stage is the determination of the most reliabe layer according to (14), with $\hat{d}_{k}=Q_{\mathrm{u}}\left\{y_{\mathrm{MMSE}, k}\right\}$ according to (23). 


\section{A. Dynamic Layer Sorting}

With (11) and (12), the maximization in (14) becomes

$k_{1}$

$=\underset{k \in\{1, \ldots, M\}}{\arg \max }\left\{\frac{\exp \left(-\left(\mathbf{y}_{\mathrm{ZF}}-\hat{d}_{k} \mathbf{e}_{k}\right)^{H} \mathbf{C}_{k}^{-1}\left(\mathbf{y}_{\mathrm{ZF}}-\hat{d}_{k} \mathbf{e}_{k}\right)\right)}{\sum_{a \in \mathcal{A}} \exp \left(-\left(\mathbf{y}_{\mathrm{ZF}}-a \mathbf{e}_{k}\right)^{H} \mathbf{C}_{k}^{-1}\left(\mathbf{y}_{\mathrm{ZF}}-a \mathbf{e}_{k}\right)\right)}\right\}$.

Using (18) and (19), this can be shown to be equivalent to

$$
k_{1}=\underset{k \in\{1, \ldots, M\}}{\arg \min } \sum_{a \in \mathcal{A}\left(\hat{d}_{k}\right)} e^{g(a, k)},
$$

where $\mathcal{A}\left(\hat{d}_{k}\right) \triangleq \mathcal{A} \backslash\left\{\hat{d}_{k}\right\}$ denotes the set of all symbols $a \in \mathcal{A}$ that are not equal to $\hat{d}_{k}$ and

$$
g(a, k) \triangleq \frac{2 \operatorname{Re}\left\{y_{\mathrm{MMSE}, k}^{*}\left(a-\hat{d}_{k}\right)\right\}-W_{k, k}\left(|a|^{2}-\left|\hat{d}_{k}\right|^{2}\right)}{1-W_{k, k}}
$$

Taking the logarithm of the expression to be minimized and applying the max-log approximation (e.g., [20]), we obtain

$$
\begin{aligned}
k_{1} & =\underset{k \in\{1, \ldots, M\}}{\arg \min }\left\{\log \left(\sum_{a \in \mathcal{A}\left(\hat{d}_{k}\right)} e^{g(a, k)}\right)\right\} \\
& \approx \underset{k \in\{1, \ldots, M\}}{\arg \min }\left\{\max _{a \in \mathcal{A}\left(\hat{d}_{k}\right)} g(a, k)\right\} .
\end{aligned}
$$

This simplifying approximation will be used in the following, and accordingly $k_{1}$ is considered to be redefined by the last expression. We can reformulate this expression in terms of the unbiased distance $\psi_{k}^{2}(\cdot)$ in $(22)$ :

$$
k_{1}=\underset{k \in\{1, \ldots, M\}}{\arg \max }\left\{\frac{W_{k, k}}{1-W_{k, k}} I_{k}\right\},
$$

with the instantaneous reliability factor (IRF)

$$
I_{k} \triangleq \min _{a \in \mathcal{A}\left(\hat{d}_{k}\right)}\left\{\psi_{k}^{2}(a)\right\}-\psi_{k}^{2}\left(\hat{d}_{k}\right)
$$

To obtain an illuminating reformulation of this result, we consider the MMSE postequalization SNR (MMSE PSNR) of the $k$ th layer (e.g., [21])

$$
\mathrm{SNR}_{k} \triangleq \frac{1}{\mathrm{MSE}_{k}}-1
$$

where $\mathrm{MSE}_{k}$ is the minimum MSE of the $k$ th layer [15], [22], i.e.,

$$
\begin{aligned}
\mathrm{MSE}_{k} & \triangleq \mathrm{E}\left\{\left|y_{\mathrm{MMSE}, k}-d_{k}\right|^{2}\right\} \\
& =\sigma_{w}^{2}\left(\left(\mathbf{H}^{H} \mathbf{H}+\sigma_{w}^{2} \mathbf{I}\right)^{-1}\right)_{k, k} \\
& =\sigma_{w}^{2}\left(\sum_{j=1}^{M} \frac{1}{\sigma_{j}^{2}+\sigma_{w}^{2}} \mathbf{v}_{j} \mathbf{v}_{j}^{H}\right)_{k, k} \\
& =\sigma_{w}^{2} \sum_{j=1}^{M} \frac{1}{\sigma_{j}^{2}+\sigma_{w}^{2}}\left|\left(\mathbf{v}_{j}\right)_{k}\right|^{2} .
\end{aligned}
$$

Here, the $\sigma_{j}^{2}$ 's and $\mathbf{v}_{j}$ 's denote, respectively, the eigenvalues and eigenvectors of $\mathbf{H}^{H} \mathbf{H}$. $\mathrm{MSE}_{k}$ can be related to $W_{k, k}$ as follows. We first note that $\mathbf{I}+\sigma_{w}^{2}\left(\mathbf{H}^{H} \mathbf{H}\right)^{-1}=\sum_{j=1}^{M}(1+$ $\left.\sigma_{w}^{2} / \sigma_{j}^{2}\right) \mathbf{v}_{j} \mathbf{v}_{j}^{H}$. From (17), we then have

$$
\begin{aligned}
W_{k, k} & =\left(\sum_{j=1}^{M} \frac{\sigma_{j}^{2}}{\sigma_{j}^{2}+\sigma_{w}^{2}} \mathbf{v}_{j} \mathbf{v}_{j}^{H}\right)_{k, k} \\
& =\sum_{j=1}^{M} \frac{\sigma_{j}^{2}}{\sigma_{j}^{2}+\sigma_{w}^{2}}\left|\left(\mathbf{v}_{j}\right)_{k}\right|^{2} \\
& =\sum_{j=1}^{M}\left|\left(\mathbf{v}_{j}\right)_{k}\right|^{2}-\sigma_{w}^{2} \sum_{j=1}^{M} \frac{1}{\sigma_{j}^{2}+\sigma_{w}^{2}}\left|\left(\mathbf{v}_{j}\right)_{k}\right|^{2} \\
& =1-\mathrm{MSE}_{k} .
\end{aligned}
$$

Inserting this into (26), we obtain $\mathrm{SNR}_{k}$ in terms of $W_{k, k}$

$$
\mathrm{SNR}_{k}=\frac{W_{k, k}}{1-W_{k, k}} .
$$

Thus, (24) can be written in terms of $\mathrm{SNR}_{k}$ as

$$
k_{1}=\underset{k \in\{1, \ldots, M\}}{\arg \max }\left\{\mathrm{SNR}_{k} I_{k}\right\} .
$$

For constant-modulus (in particular, PSK) symbol alphabets, (27) can be shown to simplify as

$$
k_{1}=\underset{k \in\{1, \ldots, M\}}{\arg \max }\left\{\mathrm{SNR}_{k} \min _{a \in \mathcal{A}\left(\hat{d}_{k}\right)} \operatorname{Re}\left\{\frac{y_{\mathrm{MMSE}, k}^{*}}{W_{k, k}}\left(\hat{d}_{k}-a\right)\right\}\right\} \text {. }
$$

\section{B. Discussion}

The quantity maximized in (27), $\mathrm{SNR}_{k} I_{k}$, is the proposed reliability measure of the detected symbol $\hat{d}_{k}=Q_{\mathrm{u}}\left\{y_{\mathrm{MMSE}, k}\right\}$ of the $k$ th layer. This quantity consists of two factors:

- The first factor is the MMSE PSNR, which expresses the average reliability of the $k$ th layer. This factor depends on the channel realization $\mathbf{H}$ and on the noise variance $\sigma_{w}^{2}$, but neither on the transmitted data vector $\mathbf{d}$ nor on the noise realization $\mathbf{w}$.

- The second factor is the IRF in (25), which can be rewritten as

$$
I_{k}=\min _{a \in \mathcal{A}\left(\hat{d}_{k}\right)}\left\{\psi_{k}^{2}(a)\right\}-\min _{a \in \mathcal{A}}\left\{\psi_{k}^{2}(a)\right\} \geq 0
$$

The IRF is seen to compare the smallest unbiased distance, $\psi_{k}^{2}\left(\hat{d}_{k}\right)=\min _{a \in \mathcal{A}}\left\{\psi_{k}^{2}(a)\right\}$, with the second smallest unbiased distance, $\min _{a \in \mathcal{A}\left(\hat{d}_{k}\right)}\left\{\psi_{k}^{2}(a)\right\}$. Thus, it expresses the instantaneous reliability of the $k$ th layer decision $\hat{d}_{k}$. It depends on $\mathbf{d}$ and $\mathbf{w}$ via $y_{\mathrm{MMSE}, k}$ (cf. (22)).

With conventional $\mathrm{NC}$, the layers are sorted simply according to maximum $\mathrm{SNR}_{k}$ or, equivalently (see (26)), according to minimum $\mathrm{MSE}_{k}$. That is, instead of (27) one has

$$
k_{1}^{\mathrm{NC}}=\underset{k \in\{1, \ldots, M\}}{\arg \max } \mathrm{SNR}_{k}=\underset{k \in\{1, \ldots, M\}}{\arg \min } \mathrm{MSE}_{k} .
$$

The new DNC layer-sorting rule (27) additionally takes into account the IRF $I_{k}$. Whereas $\mathrm{SNR}_{k}$ and $\mathrm{MSE}_{k}$ merely measure 


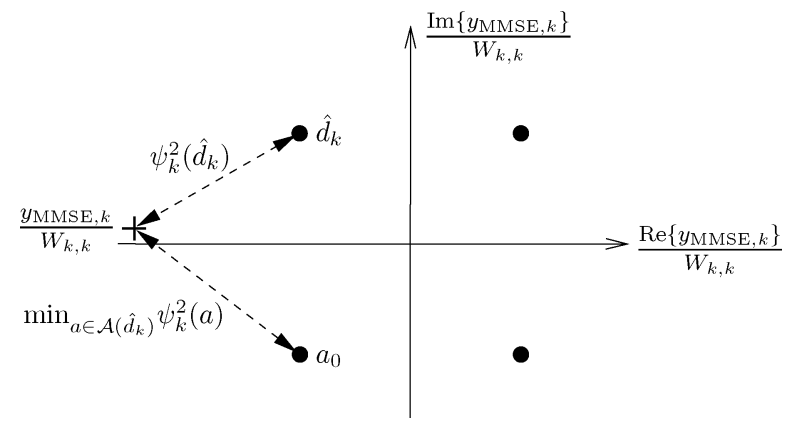

Fig. 1. Situation where the IRF can lead to a significant performance improvement of DNC over NC.

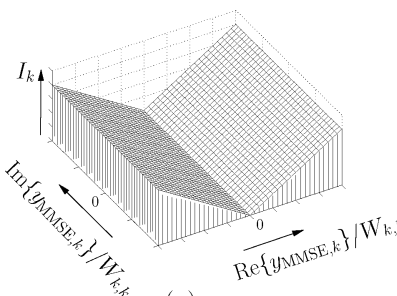

(a)

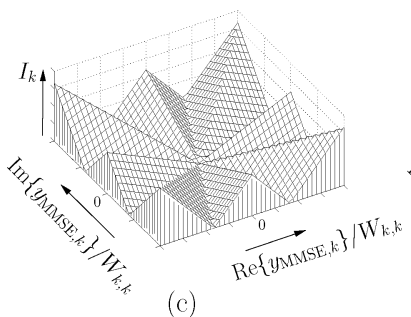

(c)

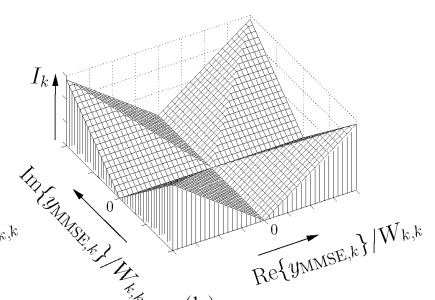

(b)

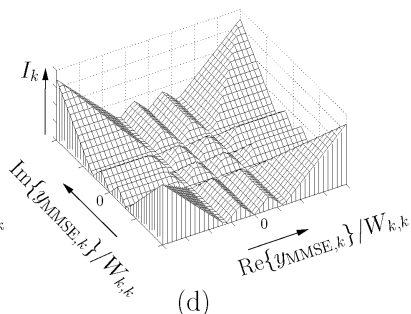

(d)
Fig. 2. IRF $I_{k}$ as a function of $y_{\mathrm{MMSE}, k} / W_{k, k}$ for the following symbol alphabets. (a) BPSK. (b) 4-QAM. (c) 8-PSK. (d) 16-QAM.

the average reliability of equalization, the IRF measures the instantaneous reliability of the subsequent detection (quantization) process. To appreciate the beneficial influence of the IRF, consider the situation shown in Fig. 1 for a 4-QAM symbol alphabet. We assume that $\mathrm{SNR}_{k}$ is large but, for a specific received vector $\mathbf{r}, y_{\mathrm{MMSE}, k} / W_{k, k}$ happens to be close to a boundary of the symbol decision regions. This means that layer $k$ is very unreliable because the unbiased distance for the detected symbol $\hat{d}_{k}$ (cf. (22)) is close to the unbiased distance for some other symbol $a_{0} \neq \hat{d}_{k}$, i.e., $\psi_{k}^{2}\left(\hat{d}_{k}\right) \approx \psi_{k}^{2}\left(a_{0}\right)$. It follows that the IRF is small, and hence DNC correctly treats this layer as unreliable. In contrast, because of the large $\mathrm{SNR}_{k}$, conventional NC would erroneously treat this layer as reliable.

In Fig. 2, the IRF is depicted as a function of $y_{\mathrm{MMSE}, k} / W_{k, k}$ for some symbol alphabets. It is seen that the IRF is a piecewise linear function of $y_{\mathrm{MMSE}, k} / W_{k, k}$. Furthermore, as discussed above, the IRF is small if $y_{\mathrm{MMSE}, k} / W_{k, k}$ is close to a boundary of the symbol decision regions; it is zero on these boundaries.

\section{THE DNC AlgorithM}

In the previous two sections, we developed the calculations of the DNC algorithm for the first decoding step. We now present a formal statement of the complete DNC algorithm, discuss its efficient implementation using a recursive matrix inversion technique, and analyze its computational complexity.

\section{A. Statement of the DNC Algorithm}

The calculations to be performed at the $l$ th decoding step of DNC, where $l \in\{1, \ldots, M\}$, are summarized below (cf. our review of conventional $\mathrm{NC}$ in Section I-D). In what follows, let $\mathbf{H}^{(l)}$ and $\mathbf{r}^{(l)}$ denote, respectively, the reduced system matrix and the interference-cleaned received vector obtained from the previous $[(l-1)$ th $]$ decoding step, with initialization according to $\mathbf{H}^{(1)}=\mathbf{H}$ and $\mathbf{r}^{(1)}=\mathbf{r}$.

1) Precalculations: Calculate the MMSE equalizer corresponding to $\mathbf{H}^{(l)}$, i.e.,

$\mathbf{G}^{(l)}=\mathbf{D}^{(l)} \mathbf{H}^{(l) H} \quad$ where $\quad \mathbf{D}^{(l)} \triangleq\left(\mathbf{H}^{(l) H} \mathbf{H}^{(l)}+\sigma_{w}^{2} \mathbf{I}\right)^{-1}$

and perform MMSE equalization

$$
\mathbf{y}_{\mathrm{MMSE}}^{(l)}=\mathbf{G}^{(l)} \mathbf{r}^{(l)} \text {. }
$$

Next, for all active layers $k=1, \ldots, M-l+1$, calculate $\mathrm{SNR}_{k}^{(l)}=\frac{1}{\mathrm{MSE}_{k}^{(l)}}-1, \quad W_{k, k}^{(l)}=1-\mathrm{MSE}_{k}^{(l)}$ where $\operatorname{MSE}_{k}^{(l)}=\sigma_{w}^{2}\left(\mathbf{D}^{(l)}\right)_{k, k}$.

2) Data detection: Perform unbiased MMSE detection for all active layers, i.e., calculate

$\hat{d}_{k}^{(l)}=Q_{\mathrm{u}}^{(l)}\left\{y_{\mathrm{MMSE}, k}^{(l)}\right\}=\underset{a \in \mathcal{A}}{\arg \min } \psi_{k}^{(l) 2}(a), k=1, \ldots, M-l+1$, with $\psi_{k}^{(l)}(a)=\left|y_{\mathrm{MMSE}, k}^{(l)} / W_{k, k}^{(l)}-a\right|$.

3) Layer sorting: Determine the most reliable layer index $k_{l} \in\{1, \ldots, M-l+1\}$ according to

$$
k_{l}=\underset{k \in\{1, \ldots, M-l+1\}}{\arg \max }\left\{\mathrm{SNR}_{k}^{(l)} I_{k}^{(l)}\right\}
$$

where $I_{k}^{(l)}=\min _{a \in \mathcal{A}\left(\hat{d}_{k}^{(l)}\right)}\left\{\psi_{k}^{(l) 2}(a)\right\}-\psi_{k}^{(l) 2}\left(\hat{d}_{k}^{(l)}\right)$.

4) Decoding and interference cancelation: Decode layer $k_{l}$ in favor of $\hat{d}_{k_{l}}^{(l)}$ and use this result for interference cancelation, i.e.,

$$
\mathbf{r}^{(l+1)}=\mathbf{r}^{(l)}-\left(\mathbf{H}^{(l)}\right)_{k_{l}} \hat{d}_{k_{l}}^{(l)}
$$

Finally, form $\mathbf{H}^{(l+1)}$ by removing from $\mathbf{H}^{(l)}$ the $k_{l}$ th column, and re-index the remaining active layers, i.e., $\{1, \ldots, M-l+1\} \backslash\left\{k_{l}\right\} \rightarrow\{1, \ldots, M-l\}$. The vector $\mathbf{r}^{(l+1)}$, matrix $\mathbf{H}^{(l+1)}$, and active-layer index set $\{1, \ldots, M-l\}$ then form the input of the next $(l \rightarrow l+1)$ decoding step.

\section{B. Recursive Calculation of $\mathbf{D}^{(l)}$}

The computational complexity of DNC is dominated by the calculation of the matrices $\mathbf{D}^{(l)}, l=1, \ldots, M$ in (29), which has to be performed anew for each received vector $\mathbf{r}$. With conventional $\mathrm{NC}$, on the other hand, layer sorting just depends on the system matrix $\mathbf{H}$, and hence the matrices $\mathbf{D}^{(l)}$ have to be calculated only once for an entire block of data vectors $\mathbf{d}$ during 
which $\mathbf{H}$ can be assumed to stay constant. For example, in the case of a spatial multiplexing system and a block-fading channel model, $\mathbf{H}$ (the MIMO channel matrix) does not change during a block of consecutive channel uses. In such cases, DNC is more complex than NC.

Fortunately, the complexity of computing the $M$ matrices $\mathbf{D}^{(l)}$ for a given received vector $\mathbf{r}$ can be significantly reduced by means of a recursive algorithm that was proposed for $\mathrm{NC}$ in [10] (see also [23]). Indeed, $\mathbf{D}^{(l+1)}$ can be calculated from $\mathbf{D}^{(l)}$ as

$$
\mathbf{D}^{(l+1)}=\left(\begin{array}{ll}
\mathbf{A}_{1} & \mathbf{A}_{2} \\
\mathbf{A}_{3} & \mathbf{A}_{4}
\end{array}\right)-\frac{1}{\alpha}\left(\begin{array}{c}
\mathbf{a}_{1} \\
\mathbf{a}_{2}
\end{array}\right)\left(\begin{array}{ll}
\mathbf{a}_{1}^{H} & \mathbf{a}_{2}^{H}
\end{array}\right)
$$

where $\mathbf{A}_{1}$ through $\mathbf{A}_{4}, \mathbf{a}_{1}$ and $\mathbf{a}_{2}$, and $\alpha$ are parts of $\mathbf{D}^{(l)}$ as shown

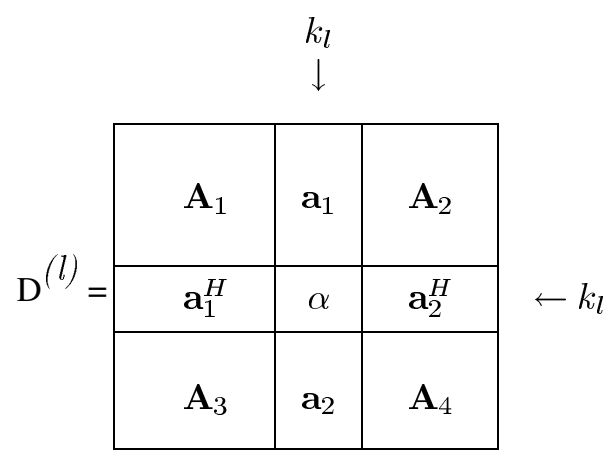

The recursion (30) is initialized by $\mathbf{D}^{(1)}=\left(\mathbf{H}^{H} \mathbf{H}+\sigma_{w}^{2} \mathbf{I}\right)^{-1}$. Whereas $\mathbf{D}^{(1)}$ depends only on $\mathbf{H}$ and $\sigma_{w}^{2}$, the subsequently calculated matrices $\mathbf{D}^{(l)}$ for $l=2, \ldots, M$ depend on the received vector $\mathbf{r}$ via $k_{l}$.

\section{Computational Complexity}

The computational complexity of the DNC algorithm can be split into the following two components:

- The "preparation complexity" $C_{\text {prep }}$ corresponds to all operations that are performed once for an entire data block during which the system matrix $\mathbf{H}$ stays constant.

- The "vector complexity" $C_{\text {vector }}$ corresponds to all operations that are performed once for each received vector $\mathbf{r}$.

For reasonable blocklengths, especially in the case of spatial multiplexing systems, $C_{\text {vector }}$ tends to dominate the overall complexity.

We will now determine estimates of $C_{\text {prep }}$ and $C_{\text {vector }}$, assuming $N=M$ for simplicity. The block complexity $C_{\text {prep }}$ is caused by the computation of the $M \times M$ matrix $\mathbf{D}^{(1)}=$ $\left(\mathbf{H}^{H} \mathbf{H}+\sigma_{w}^{2} \mathbf{I}\right)^{-1}$, and thus $C_{\text {prep }}=\mathcal{O}\left(M^{3}\right)$. The vector complexity $C_{\text {vector }}$ is composed as follows.

- The complexity of calculating the $M-1$ matrices $\mathbf{D}^{(l)}$ of size $(M-l+1) \times(M-l+1), l=2, \ldots, M$ is $\mathcal{O}\left(M^{4}\right)$ when a direct calculation is used and $\mathcal{O}\left(M^{3}\right)$ when the recursive algorithm of Section V-B is used.

- We have to perform $M$ equalization, detection, and layersorting steps for system models of size $(M-l+1) \times(M-$ $l+1), l=1, \ldots, M$; the complexity of these operations is $\mathcal{O}\left(M^{3}\right)$.

- Finally, the $M$ interference cancelation steps have a complexity of $\mathcal{O}\left(M^{2}\right)$.
Thus, the overall computational complexity of DNC using the efficient recursive calculation of the matrices $\mathbf{D}^{(l)}$ is $\mathcal{O}\left(M^{3}\right)$ per received vector.

With DNC, $C_{\text {vector }}$ is much more significant relative to $C_{\text {prep }}$ than with NC. Fortunately, the recursive calculation of the matrices $\mathbf{D}^{(l)}$ yields a strong reduction of $C_{\text {vector. The resulting }}$ overall complexity of DNC is just a fraction of the complexity of the sphere-decoding algorithm for ML detection (see Section VII-C). We note that the recursive calculation of the $\mathbf{D}^{(l)}$ 's yields larger benefits for DNC than for NC, since for NC only $C_{\text {prep }}$ is reduced. A numerical comparison of computational complexity will be presented in Section VII-C.

\section{ERror PERFormanCE OF DNC}

In Section IV-B, we argued that dynamic layer sorting based on the IRF should lead to a performance advantage of DNC over NC. We will now demonstrate this performance advantage by studying the error performance of the first layer-decoding step of DNC. The first layer-decoding step is important because it has a decisive impact on the overall error performance of $\mathrm{NC}$ schemes.

\section{A. Symbol Error Probability of a Two-Layer BPSK System}

For mathematical tractability, we consider the simple special case of two layers using BPSK modulation. The two components of $\mathbf{y}_{\text {MMSE }}$ are assumed statistically independent and Gaussian. (Our numerical simulations in Section VII-A will demonstrate that the results obtained under these simplifying assumptions are consistent with the performance observed when the assumptions are not satisfied.) The system matrix $\mathbf{H}$ is considered fixed.

For BPSK modulation, dynamic layer sorting (28) simplifies

$k_{1}=\underset{k \in\{1, \ldots, M\}}{\arg \max }\left\{\operatorname{SNR}_{k} \hat{d}_{k} z_{k}\right\}, \quad$ with $z_{k} \triangleq \frac{\operatorname{Re}\left\{y_{\mathrm{MMSE}, k}\right\}}{W_{k, k}}$

where $\hat{d}_{k}$ denotes the result of conventional MMSE detection, i.e.,

$$
\stackrel{\hat{d}_{k}}{=} Q\left\{y_{\mathrm{MMSE}, k}\right\}=\operatorname{sgn}\left(\operatorname{Re}\left\{y_{\mathrm{MMSE}, k}\right\}\right)=\operatorname{sgn}\left(z_{k}\right) \text {. }
$$

It follows that

$$
k_{1}=\underset{k \in\{1, \ldots, M\}}{\arg \max }\left\{\mathrm{SNR}_{k}\left|z_{k}\right|\right\} .
$$

To calculate the symbol error probability for the $k$ th layer, we note that

$$
z_{k}=d_{k}+n_{k}
$$

where $n_{k}$ is statistically independent of $d_{k}$. Under the Gaussian approximation for the postequalization interference, $n_{k}$ is Gaussian with zero mean and variance $\sigma_{n_{k}}^{2}=1 /\left(2 \mathrm{SNR}_{k}\right)$. Thus, the error probability of MMSE detection for the $k$ th layer is given by [24]

$$
\mathrm{P}\left[\hat{d}_{k} \neq d_{k}\right]=\mathrm{Q}\left(\sqrt{2 \mathrm{SNR}_{k}}\right)
$$

where $\mathrm{Q}(\cdot)$ denotes the $\mathrm{Q}$-function. This result is valid for both $\mathrm{NC}$ and DNC. For NC, it motivates layer sorting according to the maximum PSNR, since the decoding process for the first 
layer should be most reliable. Thus, the error probability of the first layer-decoding step of conventional $\mathrm{NC}$ is given by

$$
\mathrm{P}_{\mathrm{NC}, 1}[\mathcal{E}]=\mathrm{Q}\left(\sqrt{2 \mathrm{SNR}_{\max }}\right)
$$

where $\mathrm{SNR}_{\max } \triangleq \max _{k \in\{1, \ldots, M\}} \mathrm{SNR}_{k}$ denotes the maximum PSNR.

For DNC, calculation of the error probability cannot be based on (35) because of the dynamic layer sorting employed. It is shown in the Appendix that for two statistically independent active layers and under the Gaussian assumption, the error probability of the first layer-decoding step of DNC is given by

$$
\mathrm{P}_{\mathrm{DNC}, 1}[\mathcal{E}]=\mathrm{Q}\left(\sqrt{2\left(\mathrm{SNR}_{1}+\mathrm{SNR}_{2}\right)}\right) .
$$

\section{B. Discussion}

Comparing (36) and (37), we see that the error probability of $\mathrm{NC}$ is determined by the maximum of the two PSNRs whereas the error probability of DNC is determined by the sum of the two PSNRs. We can draw the following conclusions.

- Since $\mathrm{SNR}_{1}+\mathrm{SNR}_{2} \geq \max \left\{\mathrm{SNR}_{1}, \mathrm{SNR}_{2}\right\}$, the error probability of DNC is upper bounded by the error probability of NC:

$$
\mathrm{P}_{\mathrm{DNC}, 1}[\mathcal{E}] \leq \mathrm{P}_{\mathrm{NC}, 1}[\mathcal{E}] .
$$

- In the limiting cases $\mathrm{SNR}_{1} / \mathrm{SNR}_{2} \rightarrow \infty$ and $\mathrm{SNR}_{2} / \mathrm{SNR}_{1} \rightarrow \infty$, the two error probabilities become equal, i.e., $\mathrm{P}_{\mathrm{DNC}, 1}[\mathcal{E}] \rightarrow \mathrm{P}_{\mathrm{NC}, 1}[\mathcal{E}]$. Hence, the error performance of $\mathrm{DNC}$ is similar to that of $\mathrm{NC}$ if one of the PSNRs is very dominant. In fact, in that case DNC effectively decodes in favor of the layer with maximum PSNR, and thus it becomes equivalent to NC.

- The performance advantage of dynamic layer sorting is most significant for equal PSNRs, i.e., when $\mathrm{SNR}_{1}=\mathrm{SNR}_{2}$. Here, $\mathrm{P}_{\mathrm{NC}, 1}[\mathcal{E}]=\mathrm{Q}\left(\sqrt{2 \mathrm{SNR}_{1}}\right)$ and $\mathrm{P}_{\mathrm{DNC}, 1}[\mathcal{E}]=\mathrm{Q}\left(\sqrt{4 \mathrm{SNR}_{1}}\right)$, corresponding to a 3-dB SNR advantage of DNC over NC. In fact, for equal PSNRs, NC randomly selects some layer and thus does not exploit the possibility of layer sorting. DNC exploits this degree of freedom, and thus achieves better performance.

The simulation results in Section VII-A suggest that these conclusions are approximately true also when the simplifying assumptions under which they were derived are not satisfied.

In [25] it has been shown that for an increasing size of the system matrix, all MMSE PSNRs associated with an iid system matrix converge to the same deterministic value. In the case of spatial multiplexing systems (where the system matrix equals the channel matrix), we can thus expect that the performance advantage of DNC over $\mathrm{NC}$ is stronger for a larger number of transmit and receive antennas. For a small system, on the other hand, the variations in the PSNRs will be large, and thus there may be channel realizations for which the performance advantage of DNC is only marginal (see Section VII-A).

Finally, we expect strong average performance advantages of DNC over NC if for each realization of the system matrix $\mathbf{H}$ the
PSNRs are grouped into subsets of equal PSNRs. In such a case, NC merely performs a sorting between these subsets; within each subset a layer is randomly selected. This again corresponds to an effective loss of degrees of freedom in performing the layer sorting. Examples are the equivalent real-valued representation of spatial multiplexing systems using QAM signaling and systems using certain linear dispersion codes (see Sections VII-A and VII-B).

\section{Simulation Results}

We will now present simulation results to assess the symbol error rate (SER) performance and computational complexity of the proposed DNC technique. In our simulations, we used MIMO channels with iid Gaussian matrix entries of unit variance. The numbers of transmit and receive antennas will be denoted by $M_{\mathrm{T}}$ and $M_{\mathrm{R}}$, respectively. The channel SNR is defined as $\mathrm{SNR} \triangleq M_{\mathrm{T}} / \sigma_{w}^{2}$ (recall that $\operatorname{var}\left\{d_{k}\right\}=1$ ).

\section{A. SER Performance of Spatial Multiplexing Systems}

For a spatial multiplexing system such as V-BLAST, the system matrix $\mathbf{H}$ is the MIMO channel matrix and the size of the system model is given by the numbers of transmit and receive antennas, i.e., $M=M_{\mathrm{T}}$ and $N=M_{\mathrm{R}}$.

1) SER Performance of First Layer-Decoding Step: We first corroborate our theoretical error probability results of Section VI for a larger system that does not comply with the simplifying assumptions made in Section VI. We consider a spatial multiplexing system with $M_{\mathrm{T}}=M_{\mathrm{R}}=8$ transmit and receive antennas, 4-QAM symbol alphabet, and a channel SNR of 15 dB. In Fig. 3(a), we show the simulated SER of the first layer-decoding step for DNC and for conventional MMSE-based NC with layer sorting according to maximum PSNR. The SER is plotted versus the ratio of the largest PSNR to the second largest PSNR (this ratio is denoted as $\rho$ ). It can be seen that the performance advantage of DNC over NC is largest when the two largest PSNRs are nearly equal, i.e., for $\rho \approx 1$. In that case, DNC achieves an SER reduction by a factor of about 20 . On the other hand, the SER reduction becomes quite small when one of the PSNRs is dominant (e.g., for $\rho=3$ the SER is reduced by just a factor of about 2). This behavior is consistent with our theoretical results in Section VI.

The impact of this behavior on the average SER performance of the first layer-decoding step of course depends on the pdf of the PSNR ratio $\rho$. In Fig. 3(b), we show an estimated pdf that has been computed in the course of the simulation described above. It can be seen that small values of $\rho$ are most likely. Thus we can expect that for the first layer-decoding step, the average SER of DNC is significantly smaller than the average SER of NC. This is important as the first layer-decoding step has a decisive impact on the overall SER performance.

2) Overall SER Performance: We studied the overall SER of DNC and NC for both the complex system model (1) and the corresponding real system model (e.g., [26]). The resulting complex and real schemes will be denoted as (D)NC-C and (D)NC-R, respectively. As a performance reference, we also simulated the ML detector. 


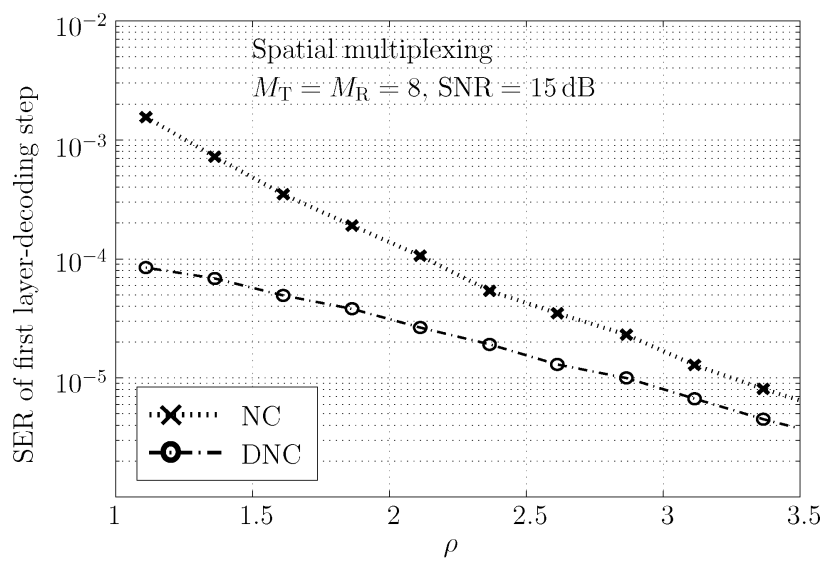

(a)

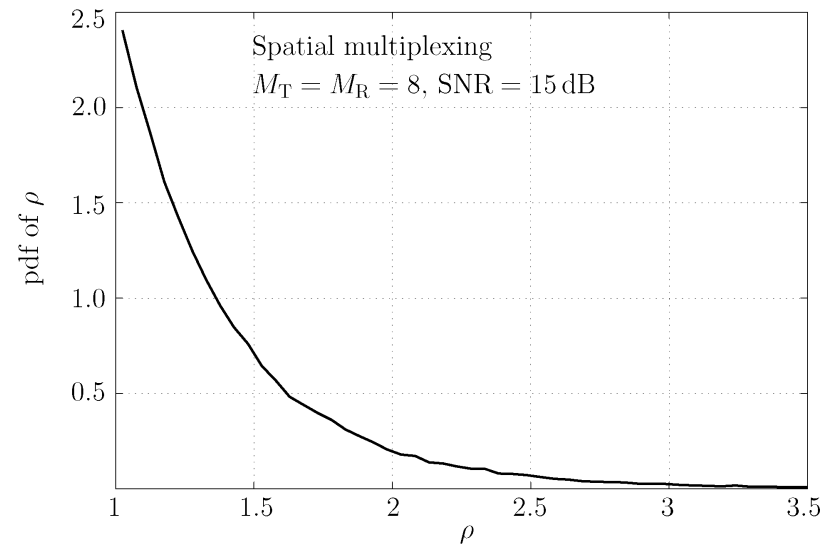

(b)

Fig. 3. Simulation results demonstrating the performance of the first layer-decoding step for conventional NC detection and the proposed DNC detector, for a spatial multiplexing system with $M_{\mathrm{T}}=M_{\mathrm{R}}=8$, 4-QAM symbol alphabet, and an SNR of $15 \mathrm{~dB}$. (a) SER versus the PSNR ratio $\rho$ (see text) corresponding to the respective channel realization. (b) Estimated pdf of $\rho$.

The real system model is given by

$$
\begin{aligned}
\left(\begin{array}{l}
\operatorname{Re}\{\mathbf{r}\} \\
\operatorname{Im}\{\mathbf{r}\}
\end{array}\right)=\left(\begin{array}{cc}
\operatorname{Re}\{\mathbf{H}\} & -\operatorname{Im}\{\mathbf{H}\} \\
\operatorname{Im}\{\mathbf{H}\} & \operatorname{Re}\{\mathbf{H}\}
\end{array}\right) & \left(\begin{array}{l}
\operatorname{Re}\{\mathbf{d}\} \\
\operatorname{Im}\{\mathbf{d}\}
\end{array}\right) \\
& +\left(\begin{array}{l}
\operatorname{Re}\{\mathbf{w}\} \\
\operatorname{Im}\{\mathbf{w}\}
\end{array}\right) .
\end{aligned}
$$

The real detection schemes detect $\operatorname{Re}\{\mathbf{d}\}$ and $\operatorname{Im}\{\mathbf{d}\}$ separately. Such a separate detection is always possible for QAM symbol alphabets-for example, a complex system with 4-QAM symbol alphabet can be reformulated as a real system of double size with BPSK symbol alphabet. For (D)NC schemes, other than for ML detection and linear equalization based detection, the complex and real implementations generally exhibit different SER performance. This is because in the real implementation, the layer sorting can be done independently for the real part and the imaginary part (see [26] for the case of conventional NC).

For a spatial multiplexing system of size $M_{\mathrm{T}}=M_{\mathrm{R}}=8$ and with 4-QAM symbols, Fig. 4(a) shows the SER-versus-SNR performance of the DNC-C and DNC-R detectors and of the various standard detectors (NC-C, NC-R, and ML). Fig. 4(b)

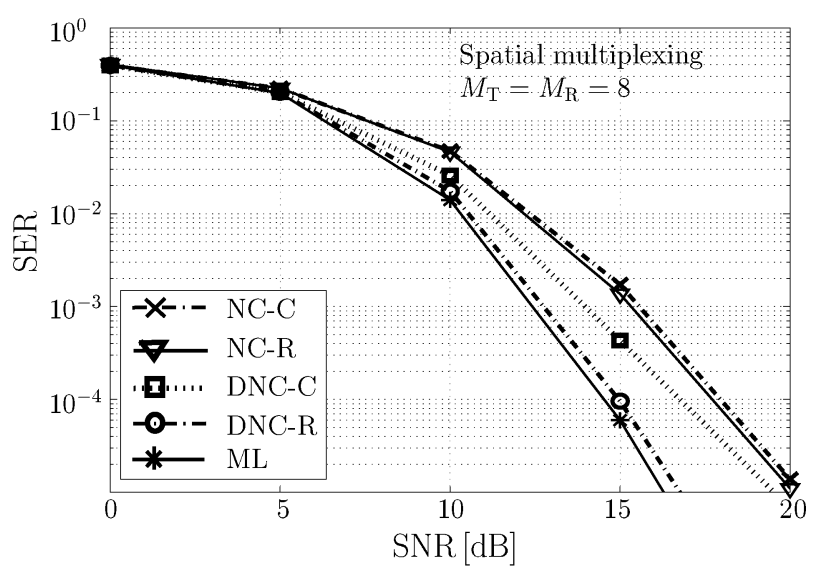

(a)

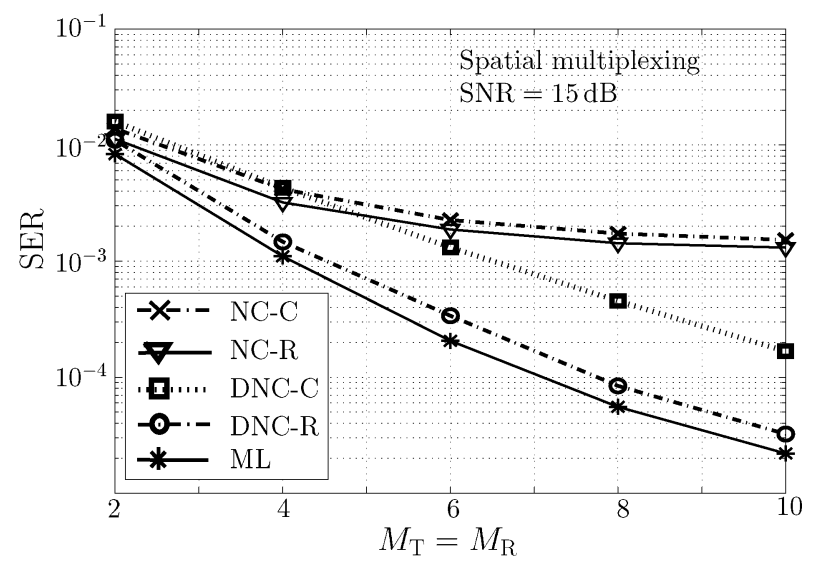

(b)

Fig. 4. SER performance of the proposed DNC detectors (DNC-C, DNC-R) and of standard detectors (NC-C, NC-R, ML) for spatial multiplexing systems using 4-QAM symbols. (a) SER versus SNR for $M_{\mathrm{T}}=M_{\mathrm{R}}=8$. (b) SER versus $M_{\mathrm{T}}=M_{\mathrm{R}}$ at an SNR of $15 \mathrm{~dB}$.

shows the SER versus $M_{\mathrm{T}}=M_{\mathrm{R}}$ at an SNR of $15 \mathrm{~dB}$. The following conclusions can be drawn from these results.

- DNC-R achieves near-ML performance over a wide range of SNRs [see Fig. 4(a)] and over a wide range of system sizes [see Fig. 4(b)].

- DNC-R performs significantly better than DNC-C. To understand this behavior, consider the case where, e.g., the real part of a given layer is reliable but the imaginary part is very unreliable (such a situation has been shown in Fig. 1). Because DNC-R performs reliability estimation and layer sorting separately for the real part and the imaginary part, it is able to correctly adapt to this situation. In contrast, DNC-C does not distinguish between the reliabilities of the real part and the imaginary part, and thus the layer sorting is the same for both parts.

- NC-R performs only slightly better than NC-C. This can be understood as follows. For NC-R, at the first decoding step, the estimated reliabilities (PSNRs) are the same for the real and imaginary parts of a given layer. Thus, the layer sorting at the first decoding step cannot be done differently for the real and imaginary parts. However, interference cancelation is performed separately, which may result in different layer orders for the real and imaginary parts in subsequent 
detection and interference cancelation steps. In contrast, with NC-C the real and imaginary parts are always canceled jointly. This explains the slight performance advantage of NC-R over NC-C (see [26] for more details).

- DNC-R performs substantially better than NC-R and NC-C. This is because DNC-R employs dynamic layer sorting which, moreover, is carried out separately for the real and imaginary parts.

- DNC-C outperforms NC-R and NC-C for $M_{\mathrm{T}}=M_{\mathrm{R}} \geq 6$.

- The performance advantage of DNC over NC is greater for larger system sizes. As explained in Section VI, for increasing system size the PSNRs of the various layers become more similar, and thus $\mathrm{NC}$ is increasingly unable to perform an appropriate layer sorting.

\section{B. SER Performance of Systems Using Linear Dispersion Codes}

Next, we consider a MIMO system using a linear dispersion (LD) code [11]. Here, the size of the system matrix (equivalent channel matrix) $\mathbf{H}$ in (1) is $N=2 M_{\mathrm{R}} L$ by $M=2 Q$, where $L$ is a blocklength and $Q$ denotes the number of data symbols transmitted during $L$ consecutive channel uses. The system model (1) is real. We used the LD code in [11, equation (31)] with $M_{\mathrm{T}}=M_{\mathrm{R}}=L=3, Q=9$, and 4-QAM symbols (here referred to as LDC1), and the LD code discussed in [11, p. 1818] with $M_{\mathrm{T}}=8, M_{\mathrm{R}}=4, L=8, Q=32$, and 16-QAM symbols (here referred to as LDC2). The size of the equivalent channel matrices associated with LDC1 and LDC2 is $18 \times 18$ and $64 \times 64$, respectively. Fig. 5(a) and (b) shows the SER-versus-SNR performance of DNC, NC, and ML detection using LDC1 and LDC2, respectively. The following conclusions can be drawn from these results.

- DNC can achieve near-ML performance. At an SER of $10^{-2}$, the SNR loss of DNC compared to ML detection is just about $0.8 \mathrm{~dB}$ for LDC1 and $1.5 \mathrm{~dB}$ for LDC2.

- DNC performs substantially better than conventional NC. This can be explained by the structure of the equivalent channel matrix $\mathbf{H}$. Indeed, at the first decoding step, the PSNRs of all layers are exactly equal for LDC1 and very similar for LDC2 (99\% of all realizations of $\mathbf{H}$ have $\rho<$ $1.5)$.

\section{Computational Complexity}

Next, we complement the complexity analysis of Section V-C by presenting empirical estimates of the computational complexity of DNC, NC, and ML detection. For spatial multiplexing systems with equal numbers of transmit and receive antennas $M_{\mathrm{T}}=M_{\mathrm{R}} \in\{4,6,8\}$ and 4-QAM symbols, Table I shows kflop estimates that were measured using MATLAB V5.3 (the corresponding SER performance was shown in Fig. 4). Even though these kflop estimates are implementation-dependent, they may be more practically meaningful than the $\mathcal{O}(\cdot)$ complexity order results presented in Section V-C. We again distinguish between the preparation complexity $C_{\text {prep }}$ (Table I(a)) and the vector complexity $C_{\text {vector }}$ (Table I(b)). Both DNC and NC-real and complex versions-were implemented using the efficient recursive matrix inversion algorithm discussed in Section V-B. For the ML detector, a straightforward

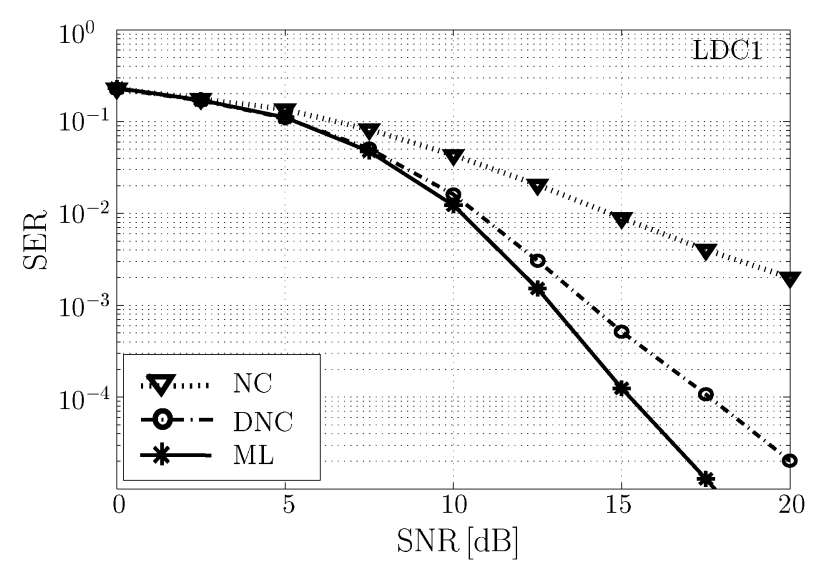

(a)

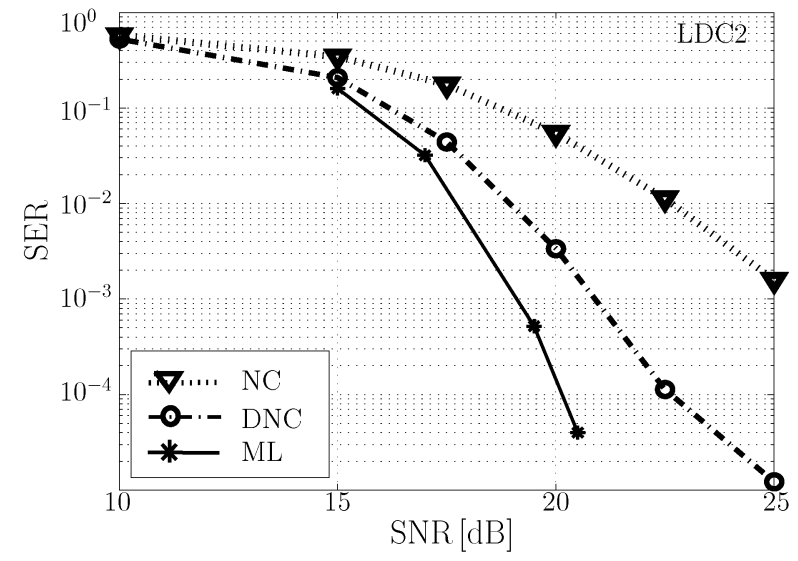

(b)

Fig. 5. SER-versus-SNR performance of the proposed DNC detector and of standard detectors for MIMO systems using LD codes. (a) First LD code (see text) with $M_{\mathrm{T}}=M_{\mathrm{R}}=3$ and 4-QAM symbols. (b) Second LD code with $M_{\mathrm{T}}=8, M_{\mathrm{R}}=4$, and 16-QAM symbols. (The SER curve for the ML detector in part (b) was adapted from [11, Fig. 9] via the approximate relation SER $\approx$ $4 \cdot$ BER.)

implementation of the sphere-decoding (SD) algorithm [4], [16], [17] was used. The complexity of SD strongly depends on the SNR and on the specific channel realization; it may exceed the average complexity of SD by a large amount. Therefore, in addition to the average SD complexity, Table $\mathrm{I}(\mathrm{b})$ shows the maximum SD complexity observed during 10000 simulation runs at an SNR of $10 \mathrm{~dB}$. The following conclusions can be drawn from Table I.

- The vector complexity $C_{\text {vector }}$ of DNC-R is just a fraction of both the average and maximum $C_{\text {vector }}$ of SD (even though DNC-R achieves near-ML performance). Note that in many applications $C_{\text {vector }}$ is the dominant complexity component.

- For DNC, $C_{\text {vector }}$ is about twice as large as for NC.

- The computational complexity of the real implementations (DNC-R, NC-R) is slightly larger than that of the complex counterparts (DNC-C, NC-C). This is due to the double system size, even though some savings are made possible by the real calculations.

- $C_{\text {prep }}$ is slightly smaller for DNC than for $\mathrm{NC}$, because with DNC a part of $C_{\text {prep }}$ is transferred to $C_{\text {vector }}$. 
TABLE I

Measured Computational Complexity (In Kflops) of the Various Detection TeChNiques for Spatial Multiplexing Systems: (a) PrePaRation COMPLEXITY $C_{\text {prep }}$, (b) VECTOR COMPLEXITY $C_{\text {vector }}$

\begin{tabular}{|c||c|c|c|c|c|}
\hline \multicolumn{6}{|c|}{$C_{\text {prep }}[$ kflops per block preparation] } \\
\hline \hline \multirow{2}{*}{$M_{\mathrm{T}}=M_{\mathrm{R}}$} & \multirow{2}{*}{$\mathrm{ML}(\mathrm{SD})$} & \multicolumn{2}{|c|}{ DNC } & \multicolumn{2}{|c|}{ NC } \\
\cline { 3 - 6 } & & $-\mathrm{R}$ & $-\mathrm{C}$ & $-\mathrm{R}$ & $-\mathrm{C}$ \\
\hline \hline 4 & 2.1 & 3.5 & 2.2 & 3.8 & 2.3 \\
\hline 6 & 6.4 & 11.3 & 6.5 & 12.2 & 7.0 \\
\hline 8 & 14.2 & 26.3 & 14.4 & 28.4 & 15.7 \\
\hline
\end{tabular}

(a)

- In contrast to SD, the complexity of DNC and NC is predetermined; it does not depend on the specific channel or noise realization.

\section{CONCLUSION}

The proposed DNC technique for MIMO detection is based on the principle that at each decoding step, the symbol and layer with maximum approximate APP are detected and canceled. The approximate APP is constructed via a Gaussian approximation for the postequalization interference. This results in an MMSE nulling technique and a "dynamic" layer-sorting rule that is superior to conventional layer sorting based on the PSNRs because it exploits the information contained in the current received vector.

The performance advantages of DNC over conventional NC were demonstrated both analytically and numerically. Specifically, we showed that the largest performance gains are obtained when the postequalization SNRs of all layers are similar. Whereas in this case NC cannot perform a meaningful layer sorting (it effectively selects some layer at random), DNC exploits the information provided by the instantaneous-reliability factor for layer sorting and thus achieves better performance. This advantage of DNC is particularly pronounced in the case of large MIMO spatial multiplexing systems and in the case of MIMO systems using certain linear dispersion codes. The additional computations required by dynamic layer sorting are strongly reduced by a recursive computation of the MMSE equalizer matrix. Our simulation results showed that DNC can yield near-ML performance for a wide range of system sizes and channel SNRs at a fraction of the computational complexity of the sphere-decoding algorithm for ML detection.

\begin{tabular}{|c||c|c|c|c|c|c|}
\hline \multicolumn{6}{|c|}{$C_{\text {vector }}$ [kflops per received vector] } \\
\hline \hline \multirow{2}{*}{$M_{\mathrm{T}}=M_{\mathrm{R}}$} & \multicolumn{2}{|c|}{ ML(SD) } & \multicolumn{2}{c|}{ DNC } & \multicolumn{2}{|c|}{ NC } \\
\cline { 2 - 7 } & av. & max. & $-\mathrm{R}$ & $-\mathrm{C}$ & $-\mathrm{R}$ & $-\mathrm{C}$ \\
\hline \hline 4 & 3.1 & 24.6 & 1.3 & 1.2 & 0.7 & 0.6 \\
\hline 6 & 13.6 & 106.2 & 4.1 & 3.3 & 2.3 & 1.5 \\
\hline 8 & 69.5 & 768 & 9.6 & 6.9 & 5.1 & 3.2 \\
\hline
\end{tabular}

(b)

\section{APPENDIX \\ Calculation of $\mathrm{P}_{\mathrm{DNC}, 1}[\mathcal{E}]$ IN (37)}

We derive the expression $\mathrm{P}_{\mathrm{DNC}, 1}[\mathcal{E}]=$ $\mathrm{Q}\left(\sqrt{2\left(\mathrm{SNR}_{1}+\mathrm{SNR}_{2}\right)}\right)$ for the error probability of the first layer-decoding step of DNC for the case of two active layers using BPSK modulation $\left(d_{1}, d_{2} \in\{-1,1\}\right)$. Let $z_{k} \triangleq \operatorname{Re}\left\{y_{\mathrm{MMSE}, k}\right\} / W_{k, k}$ as in (31). Because of symmetry, $\mathrm{P}_{\mathrm{DNC}, 1}[\mathcal{E}]$ is equal to the conditional error probability given any specific choice of transmitted symbols $d_{1}$ and $d_{2}$, e.g., $d_{1}=d_{2}=1$

$$
\mathrm{P}_{\mathrm{DNC}, 1}[\mathcal{E}]=\mathrm{P}_{\mathrm{DNC}, 1}\left[\mathcal{E} \mid d_{1}=d_{2}=1\right] .
$$

We recall from (33) and(32) that the layer-sorting and symboldetection rules are respectively given by

$$
k_{1}=\underset{k \in\{1,2\}}{\arg \max }\left\{\mathrm{SNR}_{k}\left|z_{k}\right|\right\}, \quad \hat{d}_{k_{1}}=\operatorname{sgn}\left(z_{k_{1}}\right) .
$$

An error in the first layer-decoding step occurs either if DNC decodes in favor of layer $1\left(\left|z_{1}\right| \geq\left(\mathrm{SNR}_{2} / \mathrm{SNR}_{1}\right)\left|z_{2}\right|\right)$ and makes a detection error $\left(\hat{d}_{1} \neq 1\right.$ or equivalently $\left.z_{1} \leq 0\right)$, or if DNC decodes in favor of layer $2\left(\left|z_{2}\right| \geq\left(\mathrm{SNR}_{1} / \mathrm{SNR}_{2}\right)\left|z_{1}\right|\right)$ and makes a detection error $\left(\hat{d}_{2} \neq 1\right.$ or equivalently $\left.z_{2} \leq 0\right)$. Thus, the conditional error event given that $d_{1}=d_{2}=1$ is

$$
\begin{aligned}
\mathcal{E}_{c} & =\left[\left(\left|z_{1}\right| \geq \frac{\mathrm{SNR}_{2}}{\mathrm{SNR}_{1}}\left|z_{2}\right|\right) \wedge\left(z_{1} \leq 0\right)\right] \\
& \vee\left[\left(\left|z_{2}\right| \geq \frac{\mathrm{SNR}_{1}}{\mathrm{SNR}_{2}}\left|z_{1}\right|\right) \wedge\left(z_{2} \leq 0\right)\right] .
\end{aligned}
$$

It can be shown that this is equivalent to the event $z_{1} \leq-\left(\mathrm{SNR}_{2} / \mathrm{SNR}_{1}\right) z_{2}$. Hence, (39) becomes

$$
\mathrm{P}_{\mathrm{DNC}, 1}[\mathcal{E}]=\mathrm{P}\left[z_{1} \leq-\frac{\mathrm{SNR}_{2}}{\mathrm{SNR}_{1}} z_{2} \mid d_{1}=d_{2}=1\right] .
$$

Using (34) and assuming that $z_{1}$ and $z_{2}$ are statistically independent and Gaussian, we obtain further (see equation at the bottom

$$
\begin{aligned}
\mathrm{P}_{\mathrm{DNC}, 1}[\mathcal{E}] & =\frac{1}{2 \pi \sigma_{n_{1}} \sigma_{n_{2}}} \int_{-\infty}^{\infty} \int_{-\infty}^{-\left(\mathrm{SNR}_{2} / \mathrm{SNR}_{1}\right) z_{2}} \exp \left(-\frac{1}{2}\left(\frac{z_{1}-1}{\sigma_{n_{1}}}\right)^{2}\right) \exp \left(-\frac{1}{2}\left(\frac{z_{2}-1}{\sigma_{n_{2}}}\right)^{2}\right) d z_{1} d z_{2} \\
& =\frac{1}{\sqrt{2 \pi} \sigma_{n_{2}}} \int_{-\infty}^{\infty} \mathrm{Q}\left(\frac{\frac{\mathrm{SNR}_{2}}{\mathrm{SNR}_{1}} z_{2}+1}{\sigma_{n_{1}}}\right) \exp \left(-\frac{1}{2}\left(\frac{z_{2}-1}{\sigma_{n_{2}}}\right)^{2}\right) d z_{2} \\
& =\frac{1}{\sqrt{2 \pi}} \int_{-\infty}^{\infty} \mathrm{Q}\left(\sqrt{\frac{\mathrm{SNR}_{2}}{\mathrm{SNR}_{1}}} x+\sqrt{\frac{2}{\mathrm{SNR}_{1}}}\left(\mathrm{SNR}_{1}+\mathrm{SNR}_{2}\right)\right) e^{-x^{2} / 2} d x
\end{aligned}
$$


of the previous page), where we used $\sigma_{n_{k}}=1 / \sqrt{2 \mathrm{SNR}_{k}}$. Finally, by applying the identity (e.g., [27])

$$
\frac{1}{\sqrt{2 \pi}} \int_{-\infty}^{\infty} \mathrm{Q}(\lambda x+\mu) e^{-x^{2} / 2} d x=\mathrm{Q}\left(\frac{\mu}{\sqrt{1+\lambda^{2}}}\right)
$$

we obtain $\mathrm{P}_{\mathrm{DNC}, 1}[\mathcal{E}]=\mathrm{Q}\left(\sqrt{2\left(\mathrm{SNR}_{1}+\mathrm{SNR}_{2}\right)}\right)$, which is (37).

\section{REFERENCES}

[1] D. Seethaler, H. Artés, and F. Hlawatsch, "Dynamic nulling-and-cancelling with near-ML performance," in Proc. IEEE Int. Conf. Acoustics, Speech, Signal Processing (ICASSP), Montreal, QC, Canada, May 2004, vol. IV, pp. 777-780.

[2] D. Seethaler, H. Artés, and F. Hlawatsch, "Dynamic versus conventional layer sorting for nulling-and-cancelling based MIMO detection," in Proc. EUSIPCO 2005, Antalya, Turkey, Sep. 2005.

[3] V. Tarokh, N. Seshadri, and A. R. Calderbank, "Space-time codes for high data rate wireless communications: Performance criterion and code construction," IEEE Trans. Inf. Theory, vol. 44, pp. 744-765, Mar. 1998.

[4] U. Fincke and M. Phost, "Improved methods for calculating vectors of short length in a lattice, including a complexity analysis," Math. Comp., vol. 44, pp. 463-471, Apr. 1985.

[5] P. W. Wolniansky, G. J. Foschini, G. D. Golden, and R. A. Valenzuela, "V-BLAST: An architecture for realizing very high data rates over the rich-scattering wireless channel," in Proc. URSI Int. Symp. Signals, Syst., Electron., Pisa, Italy, Sep. 1998, pp. 295-300.

[6] G. D. Golden, G. J. Foschini, R. A. Valenzuela, and P. W. Wolniansky, "Detection algorithm and initial laboratory results using V-BLAST space-time communications architecture," Electron. Lett., vol. 35, pp. 14-16, Jan. 1999

[7] W.-J. Choi, R. Negi, and J. M. Cioffi, "Combined ML and DFE decoding for the V-BLAST system," in Proc. IEEE Int. Conf. Communications (ICC), New Orleans, LA, Jun. 2000, pp. 18-22.

[8] B. Hassibi, "A fast square-root implementation for BLAST," in Proc. 34th Asilomar Conf. Signals, Syst., Comput., Pacific Grove, CA, Nov./ Dec. 2000, pp. 1255-1259.

[9] J. Luo, K. R. Pattipati, P. K. Willett, and F. Hasegawa, "Near-optimal multiuser detection in synchronous CDMA using probabilistic data association," IEEE Commun. Lett., vol. 5, pp. 361-363, Sep. 2001.

[10] J. Benesty, Y. Huang, and J. Chen, "A fast recursive algorithm for optimum sequential signal detection in a BLAST system," IEEE Trans. Signal Process., vol. 51, no. 7, pp. 1722-1730, Jul. 2003.

[11] B. Hassibi and B. Hochwald, "High-rate codes that are linear in space and time," IEEE Trans. Inf. Theory, vol. 48, no. 7, pp. 1804-1824, 2002.

[12] __ "Cayley differential unitary space-time codes," IEEE Trans. Inf. Theory, vol. 48, pp. 1485-1503, Jun. 2002.

[13] S. M. Kay, Fundamentals of Statistical Signal Processing: Detection Theory. Upper Saddle River, NJ: Prentice-Hall, 1998.

[14] G. H. Golub and C. F. Van Loan, Matrix Computations, 3rd ed. Baltimore, MD: The Johns Hopkins Univ. Press, 1996.

[15] S. M. Kay, Fundamentals of Statistical Signal Processing: Estimation Theory. Englewood Cliffs, NJ: Prentice-Hall, 1993.

[16] J. Jaldén and B. Ottersten, "An exponential lower bound on the expected complexity of sphere decoding," in Proc. IEEE Int. Conf. Acoustics, Speech, Signal Processing (ICASSP), Montreal, QC, Canada, May 2004, vol. IV, pp. 393-396.

[17] B. Hassibi and H. Vikalo, "On the sphere decoding algorithm I. Expected complexity," IEEE Trans. Signal Process., vol. 53, no. 8, pp. 2806-2818, Aug. 2005.

[18] A. Klein, G. Kaleh, and P. W. Baier, "Zero forcing and minimum mean square error equalization for multiuser detection in code-division multiple-access channels," IEEE Trans. Veh. Technol., vol. 45, pp. 276-287, May 1996.

[19] J. M. Cioffi, G. P. Dudevoir, M. V. Eyuboglu, and G. D. Forney, "MMSE decision-feedback equalizers and coding-Part I: Equalization results," IEEE Trans. Commun., vol. 43, pp. 2582-2594, Oct. 1995.

[20] S. Bäro, J. Hagenauer, and M. Witzke, "Iterative detection of MIMO transmission using a list-sequential LISS detector," in Proc. IEEE Int. Conf. Communications (ICC), Anchorage, AK, May 2003, pp. 2653-2657.
[21] R. W. Heath, S. Sandhu, and A. J. Paulraj, "Antenna selection for spatial multiplexing systems with linear receivers," IEEE Commun. Lett., vol. 5, pp. 142-144, Apr. 2001.

[22] C. W. Therrien, Discrete Random Signals and Statistical Signal Processing. Englewood Cliffs, NJ: Prentice-Hall, 1992.

[23] J. López-Vicario, C. Mecklenbräuker, and C. Antón-Haro, "Reducedcomplexity methods for throughput maximization in MIMO channels," in Proc. IEEE Int. Conf. Communications (ICC), Paris, France, Jun. 2004, vol. 5, pp. 2678-2682.

[24] J. G. Proakis, Digital Communications, 3rd ed. New York: McGrawHill, 1995.

[25] J. Evans and D. Tse, "Large system performance of linear multiuser receivers in multipath fading channels," IEEE Trans. Inf. Theory, vol. 46, no. 6, pp. 2059-2078, 2000.

[26] R. F. H. Fischer and C. Windpassinger, "Real versus complex-valued equalisation in V-BLAST systems," Electron. Lett., vol. 39, pp. 470-471, Mar. 2003.

[27] S. Verdú, Multiuser Detection. Cambridge, U.K.: Cambridge Univ. Press, 1998.

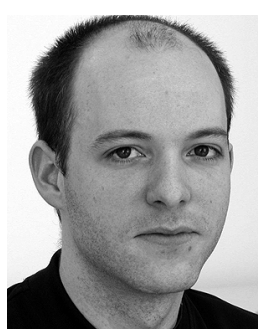

Dominik Seethaler (S'03) received the Dipl.-Ing. degree in electrical engineering from Vienna University of Technology, Vienna, Austria, in 2002.

$\mathrm{He}$ is currently a Research and Teaching Assistant with the Institute of Communications and RadioFrequency Engineering, Vienna University of Technology, where he is working toward the Ph.D. degree. His research interests are in wireless communications with emphasis on MIMO and multiuser techniques.

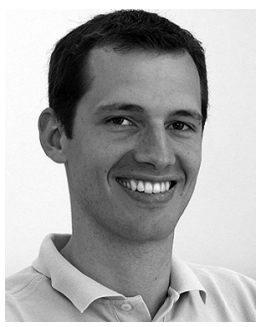

Harold Artés (S'98-M'05) received the Dipl.-Ing. and $\mathrm{Ph} . \mathrm{D}$. degrees in electrical engineering from $\mathrm{Vi}$ enna University of Technology, Vienna, Austria, in 1997 and 2003, respectively.

He was a Research (and partly Teaching) Assistant with the Institute of Communications and Radio-Frequency Engineering, Vienna University of Technology from December 1997 until February 2004. He participated in the European Commission-funded IST project ANTIUM and coauthored two patents. He is currently spending a postdoctoral year with Stanford University, Stanford, CA. He is consulting for Beceem Communications in the areas of channel estimation, synchronization, and signal processing for communications for a WiMax OFDMA system. His research interests include MIMO wireless communications, multiuser techniques, and statistical signal processing in general.

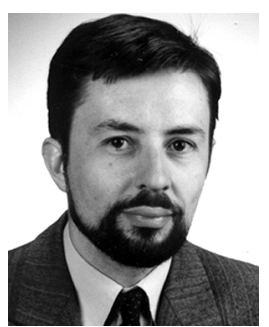

Franz Hlawatsch (S'85-M'88-SM'00) received the Diplom-Ingenieur, Dr. techn., and Univ.-Dozent (habilitation) degrees in electrical engineering/signal processing from the Vienna University of Technology, Vienna, Austria, in 1983, 1988, and 1996, respectively.

Since 1983, he has been with the Institute of Communications and Radio-Frequency Engineering, Vienna University of Technology, where he holds an Associate Professor position. During 1991-1992, as a recipient of an Erwin Schrödinger Fellowship, he spent a sabbatical year with the Department of Electrical Engineering, University of Rhode Island, Kingston, RI. In 1999, 2000, and 2001, he held one-month Visiting Professor positions with INP/ENSEEIHT/TeSA, Toulouse, France, and IRCCyN, Nantes, France. He (co-)authored a book, a review paper that appeared in the IEEE Signal Processing Magazine, about 150 refereed scientific papers and book chapters, and two patents. He coedited two books. His research interests include signal processing for wireless communications, nonstationary statistical signal processing, and time-frequency signal processing.

Prof. Hlawatsch was Technical Program Co-Chair of EUSIPCO 2004 and served on the technical committees of numerous IEEE conferences. He is currently serving as an Associate Editor for the IEEE TRANSACTIONS ON SIGNAL PROCESSING and as a member of the IEEE SPCOM Technical Committee. He is coauthor of a paper that won an IEEE Signal Processing Society Young Author Best Paper Award. 